\title{
Profiles of lipids, blood pressure and weight changes among premenopausal Chinese breast cancer patients after adjuvant chemotherapy
}

Winnie Yeo ${ }^{1,2^{*}}$, Frankie K. F. Mo ${ }^{1,2}$, Elizabeth Pang ${ }^{1,2}$, Joyce J. S. Suen ${ }^{1}$, Jane Koh ${ }^{1,2}$, Herbert H. F. Loong ${ }^{1,2}$, Christopher C. H. Yip ${ }^{1}$, Rita Y. W. Ng${ }^{1}$, Claudia H. W. Yip ${ }^{1}$, Nelson L. S. Tang ${ }^{3,4}$ and Giok S. Liem ${ }^{1}$

\begin{abstract}
Background: Adjuvant chemotherapy improves outcome of patients with early breast cancer. However, chemotherapy may be associated with long term toxicities. In this retrospective cohort study, the objectives were to determine body weight, body mass index (BMI), blood pressure and fasting lipids levels of young premenopausal Chinese breast cancer patients after adjuvant chemotherapy. Potential factors associated with these parameters were identified.

Methods: Eligibility criteria include premenopausal Chinese patients who were diagnosed to have stage I-III breast cancer within 3-10 years, age $<45$ and having received adjuvant chemotherapy at the time of breast cancer diagnosis. Information at initial breast cancer diagnosis were retrieved from patients' medical records and include age at diagnosis, tumor characteristics, anti-cancer treatments, blood pressure and body weight and height. At study entry, all patients had additional background demographics collected, as well as blood pressure, body weight and fasting serum lipid profiles measured. Incidence of chemotherapy-related amenorrhoea (CRA) and menopause were determined. Factors associated with weight gain, hypertension and dyslipidaemias were analyzed.

Results: Two hundred and eighty patients were studied. The median age at breast cancer diagnosis was 41 years (range: 24-45). The median time from breast cancer diagnosis to study entry was 5.0 years. The median age at study entry was 46.5 years (range: $28-54$ ). $91.1 \%$ developed CRA; $48.9 \%$ had become menopausal and 10\% were peri-menopausal. Between initial breast cancer diagnosis and the time of study entry, the median weight gain was $1.8 \mathrm{~kg} ; 63.2 \%$ gained weight by $>2 \%$; $52.1 \%$ were overweight/obese; $30.7 \%$ had hypertension. Abnormal total-cholesterol and LDL-cholesterol occurred in $34.3 \%$ and $56.1 \%$ respectively. On multivariate analyses, older age was associated with reduced risk while occurrence of CRA and having received taxane-containing regimens were associated with increased risk of weight gain. Oestrogen-receptor positivity was associated with reduced risk while overweight/ obese statuses were associated with increased risk of hypertension. Use of tamoxifen was associated with reduced risk of abnormal LDL-cholesterol. Weight gain, overweight/obese, older age, progression to post/peri-menopausal status at study entry, having received corticosteroid premedication before adjuvant chemotherapy and having received taxane-containing adjuvant chemotherapy were associated with increased risk of dyslipidaemias.

\footnotetext{
* Correspondence: winnieyeo@cuhk.edu.hk

'Department of Clinical Oncology, Prince of Wales Hospital, The Chinese

University of Hong Kong, Shatin, New Territories, Hong Kong SAR

${ }^{2}$ State Key Laboratory in Oncology in South China, Prince of Wales Hospital,

The Chinese University of Hong Kong, Shatin, New Territories, Hong Kong

SAR

Full list of author information is available at the end of the article
} 
(Continued from previous page)

Conclusion: Among young premenopausal Chinese breast cancer patients who had received adjuvant chemotherapy, the current study has revealed that although there was only a median weight gain of $1.8 \mathrm{~kg}$, there was a nearly 60\% increase in abnormal BMI. Further, a significant proportion of patients were detected to have hypertension and dyslipidaemias. Interventional studies with lifestyle modifications are warranted.

Keywords: Cytotoxic, Cholesterols, Dyslipidemia, Hypertension, Obesity

\section{Background}

In China, breast cancer is the most common female malignancy, with an age standardized rate of 27.96 per 100,000 population [1]; the corresponding figure is relatively higher in Hong Kong at 64.6 per 100,000 population, with over $80 \%$ of newly diagnosed breast cancer patients having diagnosed with early stage disease [2]. For women with early stage breast cancer who have undergone curative surgery, adjuvant therapies have shown to improve disease-free and overall survival. Anticancer treatment, especially cytotoxic chemotherapy, is associated with immediate as well as long-term toxicities, which may affect the quality of life and well-being of cancer survivors [3, 4]. Some of the chemotherapy-related long term toxicities include chemotherapy-related amenorrhoea (CRA) and early menopause, as well as anthropometric and metabolic changes that could lead to increased cardiovascular risk $[5,6]$.

Weight gain is a common occurrence in women diagnosed with breast cancer [7-10]. Patients who underwent adjuvant chemotherapy had reportedly gained more weight than those treated with hormonal or radio- therapies [9-12]; such difference continued to be observed over years, and a return to initial weight following weight gain was infrequent [9]. With old chemotherapeutic regimens, studies in the UK and the US had indicated that women who underwent chemotherapy gained an average of 2.5$6.7 \mathrm{~kg}$ in weight, although gains in excess of $10 \mathrm{~kg}$ had not been uncommon $[13,14]$. Using contemporary chemotherapy regimens that included taxanes as well as anthracyclines, a French study reported a mean weight gain of $3.9 \mathrm{~kg} 1$ year after treatment [15]. Apart from weight gain and obesity which is associated with detrimental effect on health profiles, anti-cancer drugs, specifically the anthracyclines and anti-human epidermal growth factor receptor 2 (HER2) agents that are commonly used in breast cancer, could cause cardiotoxicity and may further increase the cardiovascular risk of the patients concerned [16]. While change in menopausal status and increased body weight have been well-linked to the use of adjuvant chemotherapy, the association of these changes with cardiovascular comorbidities, specifically, aspects on blood pressure and lipids profiles, are less well-known.

The objectives of this retrospective cohort study on young premenopausal Chinese women with early breast cancer in Hong Kong were to (1) determine the body weight, body mass index (BMI), blood pressure and fasting lipids levels of young premenopausal breast cancer patients after adjuvant chemotherapy; and (2) identify potential factors associated with these parameters.

\section{Methods}

Between September 2008 and February 2011, eligible breast cancer patients who were attending the breast cancer follow-up clinic of the Prince of Wales Hospital were approached for study enrolment. Eligibility criteria included female of Chinese ethnicity, a history of stage I-III breast cancer within 3-10 years, premenopausal, age younger than 45 years and having received adjuvant chemotherapy at the time of breast cancer diagnosis. Patients were excluded if they had evidence of disease recurrence. Patients who received ovarian ablation as part of the endocrine therapy or had hysterectomy prior to breast cancer diagnosis were also excluded. Eligible patients identified during their follow up visits were consented for the study. The study was approved by the Joint CUHK-NTEC Clinical Research Ethics Committee of the Chinese University of Hong Kong and Hong Kong Hospital Authority.

\section{Data collection}

Clinical details at the time of breast cancer diagnosis were retrieved from individual patient's medical records. These included patient's age at breast cancer diagnosis, cancer characteristics (stage, oetrogen receptors [ER], progesterone receptors [PR] and HER2 status of the breast tumour) and information on treatment she received for her breast cancer (type of breast surgery, details of adjuvant radiotherapy, chemotherapy, tamoxifen and trastuzumab, and history of corticosteroid premedication during chemotherapy). Individual patient's blood pressure, body height and weight, measured by clinic staff at the time of diagnosis, were also retrieved from the medical records.

At study entry, patients' demographics (education level, employment history, level of family income, smoking and alcohol history, family history of breast cancer and number of live births before breast cancer diagnosis) were collected. Each patient had blood pressure and body weight measured by a research assistant. Each patient was asked to complete a study questionnaire in which they recalled 
their menstruation history with the assistance of a research assistant within the same hospital visit. In the questionnaire, patients were asked in details on the following: [1] last menstrual period (LMP) before commencement of chemotherapy; [2] after commencement of chemotherapy, presence or absence of a period of amenorrhoea with dates; [3] in case of occurrence of amenorrhoea, whether there was subsequent return of menstruation with dates; [4] for patients who had resumption of menstruation, any subsequent amenorrhoea with dates. For patients who did not experience amenorrhoea during chemotherapy, the subsequent menstrual history was further collected similarly. LMP prior to study entry was determined for each patient. Fasting blood was taken for lipid profiles, which included total-cholesterol, low density lipoprotein (LDL-) cholesterol, high density lipoprotein (HDL-) cholesterol and triglyceride levels.

\section{Definitions}

CRA was defined as amenorrhoea for $\geq 3$ months during and within 12 months after the completion of adjuvant chemotherapy [17]. Menopause was defined in line with World Health organization (WHO) criteria as 12 months of amenorrhoea with last menstrual period (LMP) $\geq 12$ months after chemotherapy and before study entry [18].

At study entry, weight gain was defined as an increase in body weight of $>2 \%$ when compared to the weight at breast cancer diagnosis [13], prior to the start of adjuvant therapies. According to the WHO criteria for Asians, BMI categories of underweight, normal weight, overweight and obesity were defined as $<18.5,18.5-<23,23-<25$, and $>/=25.0 \mathrm{~kg} / \mathrm{m} 2$ respectively [19].

Based on criteria of International Society of Hypertension, hypertension was defined as rise in systolic blood pressure (SBP) to $>/=140 \mathrm{mmHg}$, and/or increase in diastolic blood pressure (DBP) to $>/=90 \mathrm{mmHg}$ [20]. According to recommendation of the US National Cholesterol Education Program, dyslipidaemia was defined as fasting serum total-cholesterol $>/=5.2 \mathrm{mmol} / \mathrm{L}$, LDL-cholesterol $>/=2.6 \mathrm{mmol} / \mathrm{L}, \quad$ HDL-cholesterol $</=1.0 \mathrm{mmol} / \mathrm{L}$, and $/$ or triglyceride $>/=1.7 \mathrm{mmol} / \mathrm{l}[21]$.

\section{Statistical analysis}

Statistical analysis was performed by SAS version 9.3. Continuous variables were expressed as means with standard deviation or median with range as appropriate. Baseline continuous variables were compared by Student's t-test or Mann Whitney U test as appropriate, and categorical variables were compared by Chi-square test. All statistical tests were two-sided, and $p$ values $<0.05$ were regarded as significant.

Univariate logistic regression was performed to identify any potential factors associated with weight gain, hypertension and dyslipidaemias post-chemotherapy. Stepwise multivariate logistic regression analysis that included significant factors was conducted.

\section{Results}

In total, 300 breast cancer patients were approached for study entry, 14 of them declined participation. As a result, 286 patients were consented to participate in this study. Two patients failed to meet inclusion criteria as they received neo-adjuvant therapy for their stage IIIb breast cancers, four patients withdrew with the reason that they didn't have time to perform the blood tests after consent. As a result, 280 eligible patients entered the study. Table 1 shows the patients' background demographics, tumour characteristics, and anti-cancer treatments received after breast cancer diagnosis. With regards to educational level, majority $(67 \%)$ of the patients received secondary school education, $17 \%$ had primary school education and $16 \%$ had tertiary or higher level of education. On employment, 45\% were on full time employment, $16 \%$ had part-time employment and the remaining $38 \%$ were either unemployed or had retired. With respect to family income, $9.6 \%$ of patients had monthly family income less than $\mathrm{HK} \$ 5000$, 54\% had income of $\mathrm{HK} \$ 5000$ $25,000,30 \%$ had income of HK\$25000-50,000 and 7\% had income over $\mathrm{HK} \$ 50,000$.

The median age at breast cancer diagnosis was 41 years (range: 24-45). Eighty-eight had Stage I, 165 had stage II and 27 had Stage III breast cancer; 73\% were ER-positive, $67 \%$ were PR-positive and $17 \%$ were HER2 positive. Adjuvant chemotherapy regimens included anthracycline-containing (65.7\%), anthracycline-taxane containing $(26.4 \%)$, taxane containing $(1.8 \%)$ and nonanthracycline/non-taxane containing (6.1\%). Two hundred and fourteen patients also received adjuvant tamoxifen. The median time from breast cancer diagnosis to study entry was 5.04 years (range: 2.96-9.94). The median age at study entry was 46.5 years (range: $28-54$ ); eight were $\leq 35$ years, 26 were aged 36-40, 76 were aged 41-45, 146 were aged $46-50$ and 24 were aged $>50$. At the time of the study, 115 patients were still on adjuvant tamoxifen therapy.

Two hundred and fifty-five (91.1\%) had experienced CRA. At study entry, 137 patients were postmenopausal and 28 were peri-menopausal. The median age of menopause was 44 years (range: 34-52). Details of the menstrual history of all patients were described in a previous report [14].

\section{Body weight and BMI at breast cancer diagnosis and at study entry}

The median weight of patients at breast cancer diagnosis was $54.6 \mathrm{~kg}$ (range: $39.0-89.0$ ), this increased by $1.8 \mathrm{~kg}$ to $56.4 \mathrm{~kg}$ (range: $39.5-92.6$ ) at study entry. At study 
Table 1 Patients' background demographic and clinical characteristics at the time of breast cancer diagnosis $(n=280)$

No. of patients $\%$

Age at breast cancer diagnosis

$\leq 35$
$36-40$
$41-45$

Height- median; range $(\mathrm{cm})$

Weight- median; range $(\mathrm{kg})$

$\mathrm{BMI}$ at diagnosis (according to HK BMI)

Underweight $(<18.5)$

Normal (18.5-22.9)

Overweight (23.0-24.9)

Obese ( $>25)$

1 or more children born before breast cancer diagnosis

Ever smoking

Ever excessive alcohol intake (> 2 units/day)

Education

Primary

Secondary

Tertiary

Higher qualification

Employment

Retired

Unemployed

Full Time

Part Time

Family income

$<\mathrm{HK} \$ 5000$

HK\$5000-25,000

HK\$25000-50,000

HK\$50,000

1st degree relative with breast cancer

T stage

$\mathrm{T} 1$
$\mathrm{~T} 2$
$\mathrm{~T} 3$
$\mathrm{~T} 4$

Nodal Status- positive $\quad 114$

TNM staging:

Stage I

Stage II

Stage IIla

ER positive

PR positive

HER2 over-expression

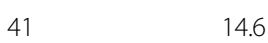

82

157

$159(141-175)$

$54.6(39.0-89.0)$

33

155

46

46

205

10

1

187

27

18

3

105

127

45
Table 1 Patients' background demographic and clinical characteristics at the time of breast cancer diagnosis $(n=280)$ (Continued)

\begin{tabular}{lll}
\hline Breast surgery: & 95 & 33.9 \\
\multicolumn{1}{l}{ Lumpectomy } & 185 & 66.1 \\
Mastectomy & 276 & 98.6 \\
Axillary lymph node dissection & 186 & 66.4 \\
Received adjuvant radiotherapy & & \\
Adjuvant chemotherapy regimen: & 17 & 6.1 \\
$\quad \begin{array}{l}\text { Non-anthracycline and non-taxane } \\
\text { containing }\end{array}$ & 184 & 65.7 \\
$\begin{array}{l}\text { Anthracycline-containing } \\
\text { Taxane-containing }\end{array}$ & 5 & 1.8 \\
$\quad$ Anthracycline- and taxane-containing & 74 & 26.4 \\
$\begin{array}{l}\text { Duration of adjuvant chemotherapy >64 days } \\
\text { Received corticosteroid premedication during }\end{array}$ & 191 & 68.2 \\
chemotherapy & 258 & 92.1 \\
Received adjuvant tamoxifen & 214 & 76.4 \\
Received adjuvant trastuzumab & 8 & 2.9 \\
$\begin{array}{l}\text { Use of traditional Chinese medicine since } \\
\text { diagnosis }\end{array}$ & 83 & 29.6 \\
\hline
\end{tabular}

entry, 177 patients (63.2\%) had gained weight by $>2 \%$, including 121 who gained $>/=5 \%$.

At study entry, $3.9 \%$ were underweight, $44.0 \%$ were normal, $22.1 \%$ were overweight, and the remaining $30.0 \%$ were obese. Eighty-five patients (30.3\%) had a shift to higher BMI categories of overweight/obese, 24 patients $(8.6 \%)$ changed from being underweight to normal, 93 (33.2\%) had maintained their BMI categories, and $78(27.6 \%)$ had decreased in their BMI categories.

\section{Incidence of hypertension at breast cancer diagnosis and at study entry}

At breast cancer diagnosis, nine patients $(3.2 \%)$ were on anti-hypertensive medications. The median SBP and DBP were 125 (range: 90-172) and $73 \mathrm{mmHg}$ (range: 42-104) respectively. Sixty-two (22.1\%) were hypertensive; 38 (13.6\%) had raised SBP, nine (3.2\%) had raised DBP and 15 (5.3\%) had raised SBP and DBP.

At study entry, 17 patients were on anti-hypertensive treatment (6.1\%). The median SBP and DBP were 127 (range: $87-206$ ) and $77 \mathrm{mmHg}$ (range: $47-128$ ) respectively. Eighty-six (30.7\%) were hypertensive; 37 (13.2\%) had raised SBP, 11 (3.9\%) had raised DBP and 38 (13.6\%) had raised SBP and DBP.

\section{Lipid profiles at study entry}

Two hundred and seventy-one patients had serum fasting lipids determined. Ninety-three patients (34.3\%) had high total-cholesterol, 152 (56.1\%) had high LDL- 
cholesterol, 18 (6.6\%) had low HDL-cholesterol and 62 (22.9\%) had hypertriglyceridaemia.

\section{Analysis for risk factors associated with weight gain, hypertension and dyslipidaemias after adjuvant chemotherapy}

Table 2 illustrates the outcomes of univariate and multivariate analyses on factors associated with weight gain. Univariate analysis revealed that older age at breast cancer diagnosis (OR 0.529, 95\% CI 0.365-0.766, $p=0.007$ ) as well as older age at study entry (OR 0.593, 95\% CI $0.429-0.819, p=0.0015)$ were both associated with less likelihood of weight gain. However, taxane-containing chemotherapy (OR 1.903, 95\% CI 1.073-3.374, $p=0.0278$ ), longer duration of adjuvant chemotherapy (OR 1.904, 95\% CI 1.137-3.189, $p=0.0143$ ), and having experienced CRA (OR 2.375, 95\% CI 1.034-5.435, $p=0.414$ ) were associated with weight gain. On multivariate analysis, taxanecontaining chemotherapy (OR 2.041, 95\% CI 1.127-3.696, $p=0.0186$ ), having experienced CRA (OR 3.472, 95\% CI 1.395-8.621, $p=0.0074$ ) and age at breast cancer diagnosis (OR for older patients 0.459, $p=0.0001$ ) were independent factors for weight gain.

Table 3 shows the outcomes of univariate and multivariate analyses on factors associated with hypertension. Univariate analysis revealed that estrogen receptor positivity

Table 2 Univariate and multivariate analysis on factors associated with weight gain $>2 \%$, by stepwise logistic regression

\begin{tabular}{|c|c|c|c|c|c|c|}
\hline & \multicolumn{3}{|c|}{$\underline{\text { Univariate analysis }}$} & \multicolumn{3}{|c|}{$\underline{\text { Multivariate analysis }}$} \\
\hline & $\mathrm{OR}$ & $95 \% \mathrm{Cl}$ for $\mathrm{OR}$ & $\mathrm{p}$ & $\mathrm{OR}$ & $95 \% \mathrm{Cl}$ for $\mathrm{OR}$ & $p$ \\
\hline Age at diagnosis & 0.529 & $0.365-0.766$ & 0.0007 & 0.459 & $0.309-0.683$ & 0.0001 \\
\hline$\leq 35$ & 1 & - & - & & & \\
\hline $36-40$ & 0.767 & $0.316-1.861$ & 0.5575 & & & \\
\hline $41-45$ & 0.332 & $0.149-0.741$ & 0.0072 & & & \\
\hline$>/=1$ children before breast cancer diagnosis & 0.751 & $0.428-1.315$ & 0.3160 & & & \\
\hline 1st degree relative with breast cancer & 1.425 & $0.488-4.167$ & 0.5173 & & & \\
\hline ER positive & 0.975 & $0.566-1.682$ & 0.9282 & & & \\
\hline PR positive & 1.211 & $0.726-2.022$ & 0.4632 & & & \\
\hline HER2 over-expression & 1.032 & $0.538-1.981$ & 0.9238 & & & \\
\hline Received adjuvant radiotherapy & 1.352 & $0.812-2.250$ & 0.2466 & & & \\
\hline Received adjuvant taxane-containing chemotherapy & 1.903 & $1.073-3.374$ & 0.0278 & 2.041 & $1.127-3.696$ & 0.0186 \\
\hline Duration of adjuvant chemotherapy $>64$ days & 1.904 & $1.137-3.189$ & 0.0143 & & & \\
\hline Received corticosteroid premedication during chemotherapy & 1.605 & $0.608-4.237$ & 0.3390 & & & \\
\hline Received adjuvant tamoxifen therapy & 1.366 & $0.778-2.398$ & 0.2781 & & & \\
\hline Received adjuvant trastuzumab & 0.969 & $0.227-4.140$ & 0.9660 & & & \\
\hline Use of traditional Chinese medicine since diagnosis & 1.303 & $0.758-2.239$ & 0.3384 & & & \\
\hline Education & 1.299 & $0.850-1.987$ & 0.2271 & & & \\
\hline Primary school & 1 & - & - & & & \\
\hline Secondary school & 1.447 & $0.757-2.766$ & 0.2642 & & & \\
\hline Tertiary school + Higher qualification & 1.669 & $0.718-3.878$ & 0.2336 & & & \\
\hline Employment- Working & 0.954 & $0.579-1.572$ & 0.8531 & & & \\
\hline Family income $</=\operatorname{HK\$ 25,000}$ & 1.388 & $0.832-2.315$ & 0.2097 & & & \\
\hline Ever Smoker & 0.374 & $0.103-1.357$ & 0.1348 & & & \\
\hline Ever excessive alcohol intake (>2 units/day) & - & - & 0.9868 & & & \\
\hline Chemotherapy-related amenorrhea & 2.375 & $1.034-5.435$ & 0.0414 & 3.472 & $1.395-8.621$ & 0.0074 \\
\hline Post/peri- menopausal at study entry & 0.666 & $0.408-1.085$ & 0.1023 & & & \\
\hline Age at study entry & 0.593 & $0.429-0.819$ & 0.0015 & & & \\
\hline$\leq 40$ & 1 & - & & & & \\
\hline $41-45$ & 0.755 & $0.297-1.923$ & 0.5561 & & & \\
\hline $46-50$ & 0.467 & $0.198-1.102$ & 0.0822 & & & \\
\hline$>50$ & 0.185 & $0.059-0.580$ & 0.0038 & & & \\
\hline
\end{tabular}


Table 3 Univariate and multivariate analyses on factors associated with hypertension at study entry, by stepwise logistic regression

\begin{tabular}{|c|c|c|c|c|c|c|}
\hline & Univari & analysis & & Multiva & e analysis & \\
\hline & OR & $95 \% \mathrm{Cl}$ for OR & p & OR & $95 \% \mathrm{Cl}$ for $\mathrm{OR}$ & $p$ \\
\hline Age at diagnosis & 1.243 & $0.869-1.779$ & 0.2343 & & & \\
\hline$\leq 35$ & 1 & - & & & & \\
\hline $36-40$ & 1.000 & $0.429-2.331$ & 1.0000 & & & \\
\hline $41-45$ & 1.417 & $0.658-3.050$ & 0.3727 & & & \\
\hline$>/=1$ children before breast cancer diagnosis & 1.291 & $0.715-2.332$ & 0.3695 & & & \\
\hline 1st degree relative with breast cancer & 0.926 & $0.316-2.715$ & 0.8885 & & & \\
\hline ER positive & 0.517 & $0.298-0.895$ & 0.0186 & 0.552 & $0.311-0.980$ & 0.0423 \\
\hline PR positive & 0.712 & $0.419-1.213$ & 0.2115 & & & \\
\hline HER2 over-expression & 1.187 & $0.610-2.309$ & 0.6134 & & & \\
\hline Received adjuvant radiotherapy & 0.866 & $0.508-1.477$ & 0.5984 & & & \\
\hline Received adjuvant taxane-containing chemotherapy & 0.887 & $0.501-1.568$ & 0.6790 & & & \\
\hline Duration of adjuvant chemotherapy $>64$ days & 0.828 & $0.483-1.420$ & 0.4928 & & & \\
\hline Received corticosteroid premedication during chemotherapy & 1.046 & $0.410-2.667$ & 0.9251 & & & \\
\hline Received adjuvant tamoxifen therapy & 0.557 & $0.313-0.990$ & 0.0462 & & & \\
\hline Received adjuvant trastuzumab & 1.352 & $0.316-5.789$ & 0.6846 & & & \\
\hline Use of traditional Chinese medicine since diagnosis & 0.947 & $0.542-1.655$ & 0.8486 & & & \\
\hline Education & 0.789 & $0.507-1.228$ & 0.2936 & & & \\
\hline Primary school & 1 & - & - & & & \\
\hline Secondary school & 0.907 & $0.461-1.787$ & 0.7784 & & & \\
\hline Tertiary school + Higher qualification & 0.609 & $0.246-1.509$ & 0.2840 & & & \\
\hline Employment- Working & 0.815 & $0.485-1.369$ & 0.4397 & & & \\
\hline Family income $</=\operatorname{HK\$ } 25,000$ & 0.753 & $0.441-1.287$ & 0.3000 & & & \\
\hline Ever Smoker & 0.271 & $0.033-2.198$ & 0.2214 & & & \\
\hline Ever excessive alcohol intake ( $>2$ units/day) & - & - & 0.9877 & & & \\
\hline Chemotherapy-related amenorrhea & 0.778 & $0.329-1.838$ & 0.5665 & & & \\
\hline Post/peri- menopausal at study entry & 0.930 & $0.559-1.548$ & 0.7808 & & & \\
\hline Weight gain $>2 \%$ at study entry & 1.095 & $0.644-1.864$ & 0.7371 & & & \\
\hline Total Cholesterol $>/=5.2 \mathrm{mmol} / \mathrm{L}$ & 1.475 & $0.865-2.516$ & 0.1534 & & & \\
\hline LDL Cholesterol >/= $2.6 \mathrm{mmol} / \mathrm{L}$ & 1.762 & $1.027-3.021$ & 0.0396 & & & \\
\hline $\mathrm{HDL}$ Cholesterol $</=1.0 \mathrm{mmol} / \mathrm{L}$ & 1.842 & $0.700-4.850$ & 0.2160 & & & \\
\hline Triglyceride $>/=1.7 \mathrm{mmol} / \mathrm{L}$ & 1.568 & $0.867-2.836$ & 0.1364 & & & \\
\hline Overweight/obese at study entry & 2.751 & $1.608-4.707$ & 0.0002 & 2.723 & $1.568-4.728$ & 0.0004 \\
\hline Age at study entry & 1.173 & $0.852-1.614$ & 0.3273 & & & \\
\hline$\leq 40$ & 1 & - & & & & \\
\hline $41-45$ & 1.929 & $0.738-5.037$ & 0.1799 & & & \\
\hline $46-50$ & 1.774 & $0.720-4.371$ & 0.2126 & & & \\
\hline$>50$ & 2.057 & $0.623-6.794$ & 0.2367 & & & \\
\hline
\end{tabular}

Hypertension defined as systolic blood pressure $>/=140 \mathrm{mmHg}$, or diastolic blood pressure $>/=90 \mathrm{mmHg}$

(OR 0.517, 95\% CI 0.298-0.895, $p=0.0186$ ) and having received adjuvant tamoxifen therapy (OR 0.557, 95\% CI $0.313-0.990, p=0.0 .0462)$ were associated with less likelihood of hypertension; while abnormal LDL-cholesterol (OR 1.762, 95\% CI 1.027-3.0221, $p=0.0396$ ) and overweight/obese at study entry (OR 2.751, 95\% CI
1.608-4.707, $p=0.0002$ ) were associated with higher likelihood of hypertension. On multivariate analysis, overweight/obese at study entry (OR 2.723, 95\% CI $1.568-4.728, p=0.0004)$ and estrogen receptor positivity (OR $0.552,95 \%$ CI $0.311-0.980, p=0.0423$ ) were independent factors for hypertension. 
Table 4 shows the factors that were identified by on univariate and multivariate analyses to be associated with dyslipidaemias. Multivariate analysis identified that older age (OR 2.093, 95\% CI 1.461-2.997, $p<0.0001$ ) and overweight/obese (OR 1.764, 95\% CI 1.013-3.073, $p=0.0451$ ) were associated with increased likelihood of high total-cholesterol. Use of tamoxifen (OR 0.342, 95\% CI 0.179-0.656, $p=0.0012$ ), overweight/obese (OR 2.560, 95\% CI 1.410-4.649, $p=0.0020$ ), older age at study entry (OR 1.625, 95\% CI 1.165-2.266, $p=0.0042$ ) and having received corticosteroids premedication during chemotherapy (OR 5.747, 95\% CI 1.264-26.32, $p=0.0236)$ were associated with high LDL-cholesterol. Becoming post/peri-menopausal (OR 3.629, 95\% CI 1.144-11.509, $p=0.0286$ ) and overweight/obese at study entry (OR 7.786, 95\% CI 1.742-34.795, $p=0.0072$ ) were associated with increased likelihood of low HDLcholesterol. Older age (OR 1.969, 95\% CI 1.258-3.017, $p=0.0019)$, taxane-containing chemotherapy (OR 2.552, 95\% CI 1.317-4.944, $p=0.0055$ ), overweight/obese (OR $2.775,95 \%$ CI $1.446-5.325, p=0.0021$ ) and weight gain (OR 2.213, 95\% CI 1.084-4.517, $p=0.0292$ ) at study entry were associated with higher likelihood of hypertriglyceridaemia. Factors that were analyzed but were not found to associate with any component of dyslipidaemias on multivariate analysis include: education level; employment and family income statuses; history of smoking and excessive alcohol intake; having child birth before breast cancer diagnosis; family history of first degree relative with breast cancer; breast ER/PR and HER2

Table 4 Factors identified to be associated with dyslipidaemia based on multivariate analysis, by stepwise logistic regression

\begin{tabular}{|c|c|c|c|c|c|c|}
\hline & \multicolumn{3}{|c|}{ Univariate analysis } & \multicolumn{3}{|c|}{ Multivariate analysis } \\
\hline & OR & $95 \% \mathrm{Cl}$ for OR & p & OR & $95 \% \mathrm{Cl}$ for OR & P \\
\hline \multicolumn{7}{|l|}{ Total cholesterol: high $(n=93)$ vs normal $(n=178)$} \\
\hline Overweight/obese at study entry & 1.760 & $1.032-3.003$ & 0.0379 & 1.764 & $1.013-3.073$ & 0.0451 \\
\hline Age at study entry & 2.101 & $1.467-3.010$ & $<0.0001$ & 2.093 & $1.461-2.997$ & $<0.0001$ \\
\hline$\leq 40$ & 1 & - & & & & \\
\hline $41-45$ & 1.490 & $0.494-4.489$ & 0.4789 & & & \\
\hline $46-50$ & 3.838 & $1.397-10.548$ & 0.0091 & & & \\
\hline$>50$ & 7.020 & $1.989-24.772$ & 0.0025 & & & \\
\hline \multicolumn{7}{|l|}{ LDL cholesterol: high $(n=152)$ vs normal $(n=115)$} \\
\hline Received corticosteroid premedication during chemotherapy & 7.576 & $1.724-33.33$ & 0.0074 & 5.747 & $1.264-26.32$ & 0.0236 \\
\hline Received adjuvant tamoxifen & 0.388 & $0.209-0.720$ & 0.0027 & 0.342 & $0.179-0.656$ & 0.0012 \\
\hline Overweight/obese at study entry & 2.468 & $1.407-4.330$ & 0.0016 & 2.560 & $1.410-4.649$ & 0.0020 \\
\hline Age at study entry & 1.626 & $1.192-2.217$ & 0.0021 & 1.625 & $1.165-2.266$ & 0.0042 \\
\hline$\leq 40$ & 1 & - & & & & \\
\hline $41-45$ & 0.963 & $0.420-2.212$ & 0.9298 & & & \\
\hline $46-50$ & 1.763 & $0.813-3.823$ & 0.1510 & & & \\
\hline$>50$ & 5.383 & $1.493-19.407$ & 0.0101 & & & \\
\hline \multicolumn{7}{|l|}{ HDL cholesterol: low ( $n=18)$ vs. normal $(n=251)$} \\
\hline Ever smoker & 4.357 & $0.836-22.707$ & 0.0806 & 7.772 & $1.102-54.827$ & 0.0397 \\
\hline Post/peri- menopausal at study entry & 3.881 & $1.243-12.114$ & 0.0196 & 3.629 & $1.144-11.509$ & 0.0286 \\
\hline Overweight/obese at study entry & 8.193 & $1.845-36.372$ & 0.0057 & 7.786 & $1.742-34.795$ & 0.0072 \\
\hline \multicolumn{7}{|l|}{ Triglyceride: high ( $n=62)$ vs. normal $(n=209)$} \\
\hline Adjuvant taxane-containing chemotherapy & 2.040 & $1.124-3.704$ & 0.0192 & 2.552 & $1.317-4.944$ & 0.0055 \\
\hline Weight gain > $2 \%$ & 2.258 & $1.171-4.355$ & 0.0150 & 2.213 & $1.084-4.517$ & 0.0292 \\
\hline Overweight/obese at study entry & 2.306 & $1.282-4.149$ & 0.0053 & 2.775 & $1.446-5.325$ & 0.0021 \\
\hline Age at study entry & 1.641 & $1.118-2.410$ & 0.0114 & 1.969 & $1.285-3.017$ & 0.0019 \\
\hline$\leq 40$ & 1 & - & & & & \\
\hline $41-45$ & 1.355 & $0.401-4.573$ & 0.6246 & & & \\
\hline $46-50$ & 2.558 & $0.842-7.773$ & 0.0978 & & & \\
\hline$>50$ & 3.733 & $0.964-14.461$ & 0.0566 & & & \\
\hline
\end{tabular}


status; having received adjuvant radiotherapy, corticosteroid or adjuvant trastuzumab; longer duration of adjuvant chemotherapy; having experienced CRA; and use of traditional Chinese medicine. Additional file 1: Table S1 lists the outcomes of univariate analyses on potential factors in association with abnormal total cholesterol, LDL-cholesterol, HDL-cholesterol ad triglyceride levels.

\section{Discussion}

Until recently, clinical follow-up of cancer survivors have mainly focused on survival and early detection of cancer recurrence. The advancement in the use of adjuvant therapies has improved outcomes of breast cancer patients, and as a result longer survivals are expected. With increased survival, it is anticipated that long-term toxicities associated with cancer treatments may become more evident; these include effects on physical morbidities and psychosocial symptoms. To our knowledge, the present study is one of the first to assess cardiovascular risk profiles of young premenopausal Chinese patients who had received modern-day adjuvant chemotherapy; apart from body weight and BMI alterations, changes on blood pressure and fasting lipids measurements have also been assessed. As the incidence of breast cancer among young Asian females has been noted to be rapidly increasing, information on these aspects among the studied population is of particular interest [22].

Although the cause of weight gaining after breast cancer diagnosis is unclear, it has been suggested that there may be ethnic differences. In the WHEL study [9], Asian-Americans were found to have a lower risk of gaining weight after chemotherapy than other races. To date, only a few studies have addressed the issue on weight changes in Asian patients. In a study from Shanghai China, breast cancer patients who received adjuvant chemotherapy had mean weight gains of 1.0, 2.0 and $1.0 \mathrm{~kg}$ at 6,18 and 36 months after diagnosis. However, details of the type and duration of chemotherapy were not described [10]. A Korean study involved 195 pre- and post-menopausal patients who received adjuvant chemotherapy (mainly anthracyclines and taxanes regimens) with/without endocrine therapy [23]. Although $10 \%$ of the studied population gained weight by $>5 \%$ at 1 year, the mean weight change was in fact, at a loss of $0.34 \mathrm{~kg}$, indicating that the majority of Korean breast cancer patients did not gain weight after adjuvant treatment. In a third study [24], 98 pre- and postmenopausal Chinese breast cancer patients underwent weight assessment prior to and immediately after having received doxorubicin and/or taxane-containing adjuvant chemotherapy; the results revealed that they had an overall mean weight loss of $0.4 \mathrm{~kg}$. The present study assessed only young premenopausal Chinese patients with a protracted interval of 5 years after adjuvant chemotherapy, $63 \%$ had gained weight by $>2 \%$ and the average gain was $1.8 \mathrm{~kg}$; although this figure appeared to be small, one cannot overlook the proportion of patients who were detected to have abnormal BMI, as this had increased by nearly $60 \%$, from $33 \%$ at breast cancer diagnosis to $52 \%$ at study entry. The figure is worrisome; according to the World Health Organization (WHO) criteria, significant health risks is associated with obesity, which has been defined as a body mass index (BMI) of 25.0 and over for Asian population [19].

A number of factors have been suggested to lead to weight gain and obesity after adjuvant chemotherapy. These include hyperphagia, younger age, menopausal status, reduced physical activity, longer duration of adjuvant chemotherapy, use of corticosteroids premedication during chemotherapy and pre-treatment body weight $[7,8,11,24-28]$. The present study has confirmed that weight gain is more common among premenopausal patients who were younger at diagnosis and who experienced CRA; in addition, those who had prior taxanecontaining regimen were also at higher risk. The latter could be associated with the use of corticosteroids during chemotherapy, as had been suggested by the study reported by Goodwin et al. [11] Dexamethasone is given as standard premedication prior to taxane therapy to avoid hypersensitivity reactions, and is also part of the optimal antiemetic regimen offered for patients on anthracyclinecontaining chemotherapy.

Only a few studies have addressed dyslipidaemias after adjuvant chemotherapy in breast cancer patients. In a recent report on Italian pre- and post-menopausal patients with early breast cancer, increase in cholesterol was found in patients who received chemotherapy only, but not in those who also received endocrine therapy [6]. The occurrence of abnormal total- and LDL-cholesterol among premenopausal patients has been attributed to chemotherapy-induced ovarian dysfunction [29-31]. The observation from a recent study lends support to this by showing that peri-menopausal patients undergoing taxane-based adjuvant chemotherapy had the largest change in lipids profiles when compared to pre- or postmenopausal patients, implicating that transition in hormone status may play a role [32]. Tamoxifen has been well-reported to lower total- and LDL-cholesterol [33-36] as early as 3 months after treatment initiation [34, 35]. Further, the administration of adjuvant tamoxifen after chemotherapy has been observed to reverse dyslipidaemias in premenopausal patients [30]. In the present study, a significant proportion of patients were found to have dyslipidaemias after chemotherapy. While tamoxifen was associated with reduced risk of abnormal LDL-cholesterol, increased likelihood of dyslipidaemias was associated with older age, post/peri-menopausal status after chemotherapy, weight gain or overweight/obese, and having received 
taxane-containing chemotherapy or corticosteroids premedication during adjuvant chemotherapy.

The present study has a few limitations; there is a lack of data on energy expenditures (e.g., physical activity levels, basal metabolic rates), caloric intakes and body composition. Further, blood pressure measurement was only performed once during clinic visit; none-the-less, this provides a glimpse on the cardiovascular status of the studied population. Dyslipidaemia may contribute to adverse cardiovascular outcomes. In this study, over 30\% of the patients were detected to be hypertensive. Multivariate analysis on risk of hypertension showed that estrogen receptor positivity was associated with decreased likelihood while overweight/obese was associated with increased likelihood of raised blood pressure. It has to be noted that additional 2 factors were identified on univariate analysis- use of tamoxifen reduced (OR 0.557, 95\% CI $0.313-0.990, p=0.0462)$ while high LDLcholesterol (OR 1.762, 95\% CI 1.027-3.021, $p=0.0396$ ) increased the likelihood of hypertension (Additional file 1: Table S1); these factors may have intertwining effects.

It has to be noted that long term toxicities of chemotherapy are not confined to menstrual disturbances, weight and BMI changes. The occurrence of hypertension and dyslipidaemias based on findings from the current study needs to be confirmed with further studies. Studies on bone health have reported that women with premature ovarian failure after adjuvant chemotherapy have increased risk for bone loss when compared to their counterparts who remain premenopausal [37, 38]. Although cognitive dysfunction among patients who developed menopause after chemotherapy has not been confirmed in earlier studies [39, 40], further assessment with improved methodology is required. Moreover, it has been shown that over 30\% of cancer survivors suffer from fatigue and sleep disturbances, anxiety, depression and impairment of physical functioning affecting their quality of life [41, 42].

\section{Conclusions}

In summary, the present study suggests that younger premenopausal patients and those who experienced CRA were more susceptible to weight gain and abnormal BMI, which in turn were associated with adverse effects on cardiovascular profiles with dyslipidemias and hypertension. Several reports have suggested that weight gain and obesity affect breast cancer outcomes [43, 44], and a recent report has implicated that high LDL-cholesterol at diagnosis [45] may also have prognostic importance. At the same time, weight gain and obesity not only affect the physical wellbeing of a patient, but have been associated with impaired quality of life among breast cancer patients after adjuvant therapy [46]. These provide good justifications for encouraging lifestyle changes to patients after breast cancer diagnosis $[6,23,47]$. In many developing countries, educational level and economical status are the major determinants of reaching healthcare facilities and preventive interventions. The lack of weight gain in Korean patients after chemotherapy has been attributed in part to patient education, which encouraged patients to maintain healthy body weight [23]. As such, data on interventional studies with lifestyle modifications to control weight and dyslipidaemia are valuable and may provide information to health care providers to better manage patients for impacts of long-term sequelae of anti-cancer treatments.

\section{Additional file}

Additional file 1: Table S1. Univariate and multivariate analysis on factors associated with abnormal total cholesterol, LDL-cholesterol, HDL-cholesterol and triglyceride; by stepwise logistic regression. Outcome of univariate and multivariate analysis on factors associated with abnormal total cholesterol, LDL-cholesterol, HDL-cholesterol and triglyceride. (DOCX $36 \mathrm{~kb}$ )

\section{Abbreviations}

BMI: Body mass index; CRA: Chemotherapy-related amenorrhea;

DBP: Diastolic blood pressure; HDL: High density lipoprotein; LDL: Low density lipoprotein; LMP: Last menstrual period; SBP: Systolic blood pressure; WHO: World Health Organization

\section{Acknowledgements}

Not applicable.

\section{Funding}

This study was supported by the Hong Kong Cancer Fund and Madam Diana Hon Fun Kong Donation for Cancer Research for the support of this study.

Availability of data and materials

The datasets analyzed during the current study will be available from the corresponding author on reasonable request.

\section{Authors' contributions}

WY contributed for the study conception, design, writing of the manuscript, acquisition of data and analysis. FKFM participated in the statistical analysis and drafting the manuscript. EP, JK, JJSS, RYWN, HHFL, CCHY, CHWY and NLST participated in the data collection and drafting the manuscript. GSL contributed for the study conception, design and writing of the manuscript. All authors read and approved the final manuscript.

Ethics approval and consent to participate

The study was approved by the Joint CUHK-NTEC Clinical Research Ethics Committee of the Chinese University of Hong Kong and Hong Kong Hospital Authority. Written consent was obtained for eligible patients at study entry.

Consent for publication

Not applicable.

Competing interests

The authors declare that they have no competing interests.

\section{Publisher's Note}

Springer Nature remains neutral with regard to jurisdictional claims in published maps and institutional affiliations.

\section{Author details}

'Department of Clinical Oncology, Prince of Wales Hospital, The Chinese University of Hong Kong, Shatin, New Territories, Hong Kong SAR. ${ }^{2}$ State Key Laboratory in Oncology in South China, Prince of Wales Hospital, The Chinese University of Hong Kong, Shatin, New Territories, Hong Kong SAR. 
${ }^{3}$ Department of Chemical Pathology, Prince of Wales Hospital, The Chinese University of Hong Kong, Shatin, New Territories, Hong Kong SAR. ${ }^{4}$ Li Ka Shing Institute of Health Sciences, Faculty of Medicine, Prince of Wales Hospital, The Chinese University of Hong Kong, Shatin, New Territories, Hong Kong SAR.

\section{Received: 25 October 2016 Accepted: 19 July 2017}

Published online: 27 July 2017

\section{References}

1. Chen W, Zheng R, Zhang S, Zeng H, Zuo T, Xia C, Yang Z, He J. Cancer incidence and mortality in China in 2013: an analysis based on urbanization level. Chin J Cancer Res. 2017;29(1):1-10.

2. Hong Kong Cancer Registry, Hospital Authority (2017) http://www3.ha.org. hk/cancereg/. Accessed 20 Apr 2017.

3. Rosenberg SM, Partridge AH. Premature menopause in young breast cancer: effects on quality of life and treatment interventions. J Thorac Dis. 2013;5:S55-61.

4. Walshe JM, Denduluri N, Swain SM. Amenorrhea in premenopausal women after adjuvant chemotherapy for breast cancer. J Clin Oncol. 2006;24:5769-79.

5. Freedman RJ, Aziz N, Albanes D, et al. Weight and body composition changes during and after adjuvant chemotherapy in women with breast cancer. J Clin Endocrinol Metab. 2004;89:2248-53.

6. Arpino G, De Angelis C, Buono G, et al. Metabolic and anthropometric changes in early breast cancer patients receiving adjuvant therapy. Breast Cancer Res Treat. 2015:154:127-32.

7. Makari-Judson G, Judson CH, Mertens WC. Longitudinal patterns of weight gain after breast cancer diagnosis: observations beyond the first year. Breast J. 2007:13:258-65.

8. Demark-Wahnefried W, Winer EP, Rimer BK. Why women gain weight after adjuvant chemotherapy for breast cancer. J Clin Oncol. 1993;11:1418-29.

9. Saquib N, Flatt SW, Natarajan L, et al. Weight gain and recovery of pre-cancer weight after breast cancer treatments: evidence from the women's healthy eating and living (WHEL) study. Breast Cancer Res Treat. 2007;105:177-86.

10. Gu K, Chen X, Zheng Y, et al. Weight change patterns among breast cancer survivors: results from the shanghai breast cancer survival study. Cancer Causes Control. 2010:21:621-9.

11. Goodwin PJ, Ennis M, Pritchard Kl, et al. Adjuvant treatment and onset of menopause predict weight gain after breast cancer diagnosis. J Clin Oncol. 1999;17:120-9

12. Demark-Wahnefried W, Peterson BL, et al. Changes in weight, body composition, and factors influencing energy balance among premenopausal breast cancer patients receiving adjuvant chemotherapy. $J$ Clin Oncol. 2001:19:2381-9.

13. Lankester KJ, Phillips JE, Lawton PA. Weight gain during adjuvant and neoadjuvant creast cancer: an audit of 100 women receiving FEC or CMF chemotherapy. Clin Ohemotherapy for bncol. 2002;14:64-7.

14. Camoriano JK, Loprinzi $\mathrm{CL}$, Ingle JN, et al. Weight change in women treated with adjuvant therapy or observed following mastectomy for node-positive breast cancer. J Clin Oncol. 1990:8:1327-34.

15. Trédan O, Bajard A, Meunier A, et al. Body weight change in women receiving adjuvant chemotherapy for breast cancer: a French prospective study. Clin Nutr. 2010;29:187-91.

16. Jones AL, Barlow M, Barrett-Lee PJ, Canney PA, Gilmour IM, Robb SD, Plummer CJ, Wardley AM, Verrill MW. Management of cardiac health in trastuzumab-treated patients with breast cancer: updated United Kingdom National Cancer Research Institute recommendations for monitoring. $\mathrm{Br} J$ Cancer. 2009;100:684-92

17. Liem GS, Mo FK, Pang E, et al. Chemotherapy-related amenorrhea and menopause in young Chinese breast cancer patients- analysis on incidence, risk factors and serum hormone profiles. PLoS One. 2015;10:e0140842.

18. World Health Organization. Technical Report Series: Research on the Menopause. Geneva: WHO Technical Report Series No. 670; 1981.

19. World Health Organization, International Association for the Study of Obesity, International Obesity Task Force. The Asia-Pacific perspective: redefining obesity and its treatment. Melbourne: Health Communications Australia Pty Ltd; 2000

20. Weber MA, Schiffrin EL, White WB et al Clinical practice guidelines for the management of hypertension in the community: a statement by the American Society of Hypertension and the International Society of Hypertension. J Clin Hypertens (Greenwich). 2014;16:14-26.
21. Grundy SM, Cleeman Jl, Merz CN, et al. Implications of recent clinical trials for the National Cholesterol Education Program Adult Treatment Panel III guidelines. J Am Coll Cardiol. 2004;44:720-32.

22. Shen YC, Chang CJ, Hsu C, et al. Significant difference in the trends of female breast cancer incidence between Taiwanese and Caucasian Americans. Cancer Epidemiol Biomark Prev. 2005;14:1986-90.

23. Han HS, Lee KW, Kim JH, et al. Weight changes after adjuvant treatment in Korean women with early breast cancer. Breast Cancer Res Treat. 2009:114:147-53.

24. Wang JS, Cai H, Wang CY, et al. Body weight changes in breast cancer patients following adjuvant chemotherapy and contributing factors. Mol Clin Oncol. 2014;2:105-10.

25. Irwin ML, McTiernan A, Baumgartner RN, et al. Changes in body fat and weight after a breast cancer diagnosis: influence of demographics, prognostic, and lifestyle factors. J Clin Oncol. 2005:23:774-82.

26. Schwartz AL. Exercise and weight gain in breast cancer patients receiving chemotherapy. Cancer Pract. 2000;8:231-7.

27. Holmes MD, Chen WY, Feskanich D, et al. Physical activity and survival after breast cancer diagnosis. JAMA. 2005;293:2479-86.

28. Rock CL, Flatt SW, Newman V, et al. Factors associated with weight gain in women after diagnosis of breast cancer. Women's healthy eating and living study group. J Am Diet Assoc. 1999;99:1212-21.

29. Saarto T, Blomqvist C, Ehnholm C, et al. Effects of chemotherapy-induced castration on serum lipids and apoproteins in premenopausal women with node-positive breast cancer. J Clin Endocrinol Metab. 1996;81:4453-7.

30. Vehmanen L, Saarto T, Blomqvist C, et al. Tamoxifen treatment reverses the adverse effects of chemotherapy-induced ovarian failure on serum lipids. $\mathrm{Br}$ J Cancer. 2004:91:476-81.

31. Gordon AM, Hurwitz S, Shapiro CL, et al. Menopause. Premature ovarian failure and body composition changes with adjuvant chemotherapy for breast cancer. Menopause. 2011;18:1244-8.

32. Kim SB, Kok YT, Thuan TV, et al. Safety results of Docetaxel-(Taxotere ${ }^{\circledR}$ )-based chemotherapy in early breast cancer patients of Asia-Pacific region: AsiaPacific breast initiative II. J Breast Cancer. 2015;18:356-64.

33. Dewar JA, Horobin JM, Preece PE, et al. Long term effects of tamoxifen on blood lipid values in breast cancer. BMJ. 1992;305:225-6.

34. Thangaraju M, Kumar K, Gandhirajan R, et al. Effect of tamoxifen on plasma lipids and lipoproteins in postmenopausal women with breast cancer. Cancer. 1994;73:659-63.

35. Ilanchezhian S, Thangaraju M, Sachdanandam P. Plasma lipids and lipoprotein alterations in tamoxifen treated breast cancer women in relation to the menopausal status. Cancer Biochem Biophys. 1995;15:83-90.

36. Joensuu $\mathrm{H}$, Holli $\mathrm{K}$, Oksanen $\mathrm{H}$, et al. Serum lipid levels during and after adjuvant toremifene or tamoxifen therapy for breast cancer. Breast Cancer Res Treat. 2000:63:225-34.

37. Shapiro CL, Manola J, Leboff M. Ovarian failure after adjuvant chemotherapy is associated with rapid bone loss in women with early-stage breast cancer. J Clin Oncol. 2001;19:3306-11.

38. Bruning PF, Pit MJ, de Jong-Bakker $M$, et al. Bone mineral density after adjuvant chemotherapy for premenopausal breast cancer. Br J Cancer. 1990;61:308-10.

39. Hermelink K, Henschel V, Untch M, et al. Short-term effects of treatmentinduced hormonal changes on cognitive function in breast cancer patients: results of a multicenter, prospective, longitudinal study. Cancer. 2008;113:2431-9.

40. Jenkins $V$, Shilling $V$, Deutsch $G$, et al. A 3-year prospective study of the effects of adjuvant treatments on cognition in women with early stage breast cancer. Br J Cancer. 2006;94:828-34.

41. Cella D, Davis K, Breitbart W, Gregory Curt for the Fatigue Coalition. Cancer-related fatigue: prevalence of proposed diagnostic criteria in United States sample of a United States sample of cancer survivors. J Clin Oncol. 2001;19:3385-91.

42. Kluthcovsky ACGC, Urbanetz AA, Carvalho DS, Maluf EMCP, Sylvestre GCS. Fatigue after treatment in breast cancer survivors: prevalence, determinants and impact on health-related quality of life. Support Care Cancer. 2012;20(8):1901-9.

43. Kroenke $\mathrm{CH}$, Chen WY, Rosner $\mathrm{B}$, et al. Weight, weight gain, and survival after breast cancer diagnosis. J Clin Oncol. 2005;23:1370-8.

44. Nichols HB, Trentham-Dietz A, Egan KM, et al. Body mass index before and after breast cancer diagnosis: associations with all-cause, breast cancer, and cardiovascular disease mortality. Cancer Epidemiol Biomark Prev. 2009;18:1403-9. 
45. Rodrigues Dos Santos C, Fonseca I, Dias S, et al. Plasma level of LDL-cholesterol at diagnosis is a predictor factor of breast tumor progression. BMC Cancer. 2014;14:132.

46. Fang $\mathrm{P}$, Tan $\mathrm{KS}$, Troxel AB, et al. High body mass index is associated with worse quality of life in breast cancer patients receiving radiotherapy. Breast Cancer Res Treat. 2013;141:125-33.

47. Rock CL, Flatt SW, Byers TE, et al. Results of the exercise and nutrition to enhance recovery and good health for you (ENERGY) trial: a behavioral weight loss intervention in overweight or obese breast cancer survivors. J Clin Oncol. 2015;33:3169-76.

Submit your next manuscript to BioMed Central and we will help you at every step:

- We accept pre-submission inquiries

- Our selector tool helps you to find the most relevant journal

- We provide round the clock customer support

- Convenient online submission

- Thorough peer review

- Inclusion in PubMed and all major indexing services

- Maximum visibility for your research

Submit your manuscript at www.biomedcentral.com/submit
Biomed Central 